\title{
Improving the care of children with urinary tract infection: use of a clinical decision proforma
}

Daniel Leach

Pennine Acute Hospitals NHS Trust

\begin{abstract}
Urinary tract infection (UTI) is a common and important clinical problem in children. Follow up imaging is indicated in some cases to reduce the risk of long-term harm from UTI and sometimes to help guide acute management. Overuse of imaging may be harmful due to radiation exposure, as well as increasing demand on services and budgets. On the other hand under-use of imaging may leave children vulnerable to renal damage and long-term morbidity. Accepted standards propose an imaging strategy specific to age and type of UTI. The complexity of the guideline makes compliance with the standards challenging.

The aim of this project was to assess current practice for imaging of children with UTI managed at The Royal Oldham Hospital and to improve compliance with accepted standards through the use of a proforma to aid clinical decision making, supported by an education programme. A retrospective audit was performed over a 6 month period both prior to and after the intervention. The baseline audit found $57.7 \%$ of children treated for UTI $(n=26)$ had imaging compliant with the accepted standards, which improved to $75.9 \%(n=29)$ on post-measurement. The percentage of inappropriate investigations reduced from $52.4 \%$ to $10.5 \%$. The percentage of missed investigations reduced from $35.0 \%$ to $32.0 \%$. The proforma was used and filed in $40 \%$ of cases where practice was in line with accepted standards. It was not used in any of the cases where practice deviated.
\end{abstract}

In conclusion, a clear clinical decision aid, supported by an education programme, can significantly improve compliance with accepted standards for imaging of children with UTI. This may also be transferable to other scenarios where guidelines exist but have reduced efficacy due to complexity and/or lack of understanding.

\section{Problem}

Although clear guidelines exist to guide the choice of imaging performed for children with urinary tract infection (UTI) there is very poor compliance in clinical practice. At The Royal Oldham Hospital (part of The Pennine Acute Hospitals NHS Trust, UK) we identified this problem initially due to the number of inappropriate imaging requests, and the lack of requests for indicated imaging that appeared to be a recurring theme when completing discharge summaries. Informal assessment of knowledge of the guidance at clinical handover sessions revealed that most were aware that guidance existed, however the majority of clinicians either missed imaging or selected inappropriate imaging in response to multiple choice questions for a given scenario.

\section{Background}

UTI is a common and important clinical problem in children. Around 1 in 10 girls and 1 in 30 boys will have had a UTI by the age of 16 years. Early recognition and appropriate management can prevent deterioration and reduce the incidence of long-term sequelae. Aside from satisfactory resolution of the acute infection, an important consideration in the management of UTI is the use of imaging to visualise the kidneys and urinary tract. The primary purpose of this imaging is to identify anatomical abnormalities such as obstructive uropathies or vesicoureteric reflux. Early identification of such abnormalities allows management such as surgery or antibiotic prophylaxis to prevent renal damage. Preventing renal damage in these situations may reduce the subsequent development of serious conditions relating to renal scarring including hypertension and renal failure.[1]

Due to the large numbers of children who are diagnosed with UTI, follow up imaging is not indicated in all cases in order to reduce the burden this would place on imaging services and the unnecessary radiation exposure that would be involved. Balancing the importance of identifying abnormalities against the clinical risk of imaging and economic burden is clearly a delicate task. The National Institute for Health and Care Excellence (NICE) has produced a guideline to support clinicians in this area. The NICE clinical guideline 54: Urinary tract infection in children (CG54)[1] was issued in 2007 and provides an imaging strategy which takes into account the age of the child, and defines recurrent and atypical UTI which require different imaging follow up.

\section{Baseline measurement}

An initial retrospective audit to establish a baseline was performed looking at 32 cases of children admitted with UTI to the Royal Oldham Hospital over a 6 month period in 2013. 6 cases were excluded (criteria for exclusion: inappropriately coded admission; not managed under a paediatric consultant; already under investigation for recurrent UTI) leaving a total of 26 cases. This 
included children in all three of the age brackets defined by the NICE guidance (age $<6$ months, $n=6$; age 6 months to 3 years, $n=9$; age $>3$ years, $n=11$ ), and included at least one case from each category of UTI defined by NICE (typical, $n=18$; atypical, $n=7$; recurrent, $n=1$ ). Each case was assessed against the criteria for atypical and recurrent UTI which, together with the age of each patient, allowed the optimal imaging strategy to be identified from the guidance. This was then compared with the imaging strategy actually performed.

Overall $57.7 \%$ of children ( 15 of 26 ) had imaging according to the accepted standards $(50.0 \%$ of children age $<6$ months ( 3 of 6 ); $44.4 \%$ of children age 6 months to 3 years ( 4 of 9 ); $72.7 \%$ of children age $>3$ years ( 8 of 11$)$ ). $52.4 \%$ of the imaging investigations performed (11 of 21 ) were inappropriate. $35.0 \%$ of the indicated imaging investigations (7 of 20) were missed. The most common error was performing an outpatient ultrasound scan within 6 weeks of the acute infection where this was not indicated. The full compliance results are attached as a supplementary file.

See supplementary file: ds4950.pdf - "Results from the baseline audit showing compliance for each of the accepted standards, split by age and UTI type"

\section{Design}

It seemed clear that to meet the accepted standards a tool would be required to support the decision making process. To identify the optimal imaging strategy the clinician must assess the patient against 7 criteria for atypical UTI, 3 criteria for recurrent UTI, and then choose from a combination of 4 different investigations. For example; an atypical UTI in a child aged $<6$ months should result in an ultrasound scan during the acute infection, a dimercaptosuccinic acid scan 4-6 months later, and an micturating cystourethrogram; whereas an atypical UTI in a child aged $>3$ years only requires an ultrasound scan during the acute infection. To support this decision making process we devised a proforma which guided the clinician firstly in correctly classifying the UTI as typical, atypical, or recurrent, and secondly in selecting the correct imaging strategy for each classification in each age range. The simple flow chart design of the proforma was designed to make the decision making process clear and simple.

However well the proforma was designed it would not impact practice if not used. To support the intervention we presented the finding of the baseline audit to the department and used this as a rationale for adopting the proforma. The importance of appropriate imaging in UTIs was included in the clinical induction programme with emphasis on using the proforma to support decision making.

\section{Strategy}

PDSA (Plan, Do, Study, Act) cycle 1

As discussed above our initial planning revolved around identifying the cause of the poor compliance identified by the baseline audit. We suggested that the complexity of the guidelines together with the need to remember a large number of different criteria and management options was a significant barrier to best practice. A proforma combined the roles of aide memoir and decision guide. The design of the proforma was important - it had to be clear and simple to encourage uptake, but still contain all the information required. We decided a flow chart with clear YES or NO branches at each decision point would provide the greatest clarity. We assessed the initial design of the proforma by asking for informal feedback from clinicians who would be using it. The response was generally positive, but they suggested that the addition of tick-boxes would make completing the proforma more attractive, as opposed to simply referring to it. It also had the additional benefit of improving accountability and recording the decision making process.

\section{PDSA cycle 2}

We adjusted the proforma to reflect the feedback from the first cycle. We decided to test it next in a non-clinical setting using example scenarios at a departmental audit meeting. A set of scenarios were provided and clinicians asked to specify the optimal imaging strategy for each, first without the benefit of the proforma, and then with. Without the proforma the majority of clinicians made at least one error per scenario. With the proforma there were no errors. The feedback was positive, although senior clinicians suggested that there should be a section added to allow a rationalisation for alternative imaging strategy to be documented on a case by case basis.

PDSA cycle 3

Based on the feedback from the audit meeting we again adjusted the proforma with the aim of maximising uptake once it was rolled out. When the proforma was used it appears from our early testing to dramatically improve compliance with the accepted standards. Evidently encouraging clinicians to use the proforma would be important for real world use. We identified 3 areas where this could be tackled; inclusion on the clinical induction for doctors new to the department and the weekly departmental teaching programme, and revisiting the topic by completing future audit loops and presenting the result. Specific named individuals were assigned to these roles to ensure accountability and a review date was set. This strategy was submitted to the trust audit department who supported the project. The proforma was then rolled out for use across the Children's inpatient and admissions areas. Post-measurement was carried out for a six month period starting six weeks after the initial roll out.

\section{Results}

The post-measurement was carried out as a retrospective audit looking at 34 cases of children admitted with UTI to the Royal Oldham Hospital over a 6 month period in 2014. 5 cases were excluded (criteria for exclusion: inappropriately coded admission; not managed under a paediatric consultant; already under investigation for recurrent UTI) leaving a total of 29 cases. This included children in all three of the age brackets defined by the NICE guidance (age $>6$ months, $n=9$; age 6 months to 3 years, $n=$ 
6 ; age $>3$ years, $n=14$ ), and included at least one case from each category of UTI defined by NICE (typical, $n=20$; atypical, $n=8$; recurrent, $n=1$ ). Each case was assessed against the criteria for atypical and recurrent UTIs, which together with the age of each patient allowed the ideal imaging strategy to be identified from the guidance. This was then compared with the imaging strategy actually performed.

Prior to the introduction of the proforma $57.7 \%$ of children treated for UTI (15 of 26) had imaging according to the accepted standards. After the proforma was introduced this improved to $75.9 \%$ (22 of 29). The percentage of imaging investigations performed that were inappropriate reduced from $52.4 \%$ (11 of 21 ) to $10.5 \%$ (2 of 19 ). The percentage of indicated imaging investigations that were missed reduced from $35.0 \%$ (7 of 20 ) to $32.0 \%$ (8 of 25 ). The proforma was used and filed in $40 \%$ of cases where practice was in line with accepted standards. It was not used in any of the cases where practice was not in line with accepted standards. It is not possible to assess cases where the proforma may have been used, but not filed in the notes. The full compliance results are attached as a supplementary file.

See supplementary file: ds4951.pdf - "Results from the post measurement audit showing compliance for each of the accepted standards, split by age and UTI type"

\section{Lessons and limitations}

Although the interventions described resulted in a dramatic improvement in the imaging strategies used for the investigation of children with UTI there is still a proportion of patients who are exposed to unnecessary investigation and, of greater concern, patients who miss out on investigations which could identify serious renal conditions and allow early intervention to reduce future morbidity. When the proforma is used it clearly has a very significant impact; this emphasises the importance of increasing uptake of the proforma. Going forward the biggest challenge this project faces is maintaining momentum and interest in using the proforma.

The project benefited from a long audit period ( 6 months) for both the baseline and post-measurement phases. However potential limitations due to the retrospective nature of the audits include missing cases which were incorrectly coded and therefore not included on the population list, or cases where criteria which may have resulted in classification of a patient into either atypical or recurrent were not recorded in notes. Another potential limitation is that clinicians may have been aware of the guidelines but decided to override them for a clinical reason which was not recorded in the notes. Since these limitations were present for both the baseline and post-measurement audit it is unlikely they would have significantly affected the results or conclusions about the impact of the intervention.

This is a cost effective intervention due to the lack of expense of producing and using the proforma, and integrating the supportive educational strategy into pre-existing protected time. However, maintaining the improvement will require sustained input to ensure that staff turnover does not erode the gains achieved. The involvement of permanent consultant grade doctors on the project team should help reduce this by maintaining continuity.

\section{Conclusion}

Instituting the clinical decision proforma and its supporting educational programme was an extremely positive action as it has significantly improved the management of children with UTI at our unit. There was a reduction in the percentage of inappropriate imaging performed and indicated imaging missed. This means that the risk of iatrogenic harm is less, and the opportunities to prevent long-term morbidity are greater. A complex guideline is not optimised for the clinical environment and is not the most appropriate method to support decision making at the bedside. The introduction of the proforma has clearly had a significantly beneficial effect, but it is important to focus on increasing the awareness and uptake of the proforma to further improve patient care. The interventions from this project may be transferable to other scenarios where guidelines exist but have reduced efficacy due to complexity and/or lack of understanding.

\section{References}

1. National Collaborating Centre for Women's and Children's Health. NICE Clinical Guideline 54: Urinary tract infection in children: diagnosis, treatment and long-term management. London: National Institute for Health and Care Excellence; 2007

\section{Declaration of interests}

Nothing to declare

\section{Acknowledgements}

Dr B. Padmakumar, Consultant Paediatrician; Dr E. Odeka, Consultant Paediatrician; Christopher Hoare, Clinical Audit Facilitator; Danielle Gregg, Audit Officer - Advancing Quality; All of Pennine Acute Hospitals NHS Trust

\section{Ethical approval}

This project has been conducted according to the ethics guidelines of The Pennine Acute Hospitals NHS Trust. The project met the following criteria for quality improvement activities exempt from ethics review; the purpose of the project was to improve the quality of patient care, care was measured against accepted standards (the project was not used to generate new knowledge as to what best practice is), and the project did not involve any change to patient care beyond routine clinical management. 